\title{
Formation of Diffusion Pairs as an Initial Stage in the Process of Decomposition of Alloys
}

\author{
Yuri Ustinovshikov \\ Udmurt Federal Research Center, Russian Academy of Sciences, Izhevsk, Russia \\ Email: ust@udman.ru
}

How to cite this paper: Ustinovshikov, Y. (2020) Formation of Diffusion Pairs as an Initial Stage in the Process of Decomposition of Alloys. Advances in Materials Physics and Chemistry, 10, 328-338. https://doi.org/10.4236/ampc.2020.1012026

Received: November 30, 2020

Accepted: December 26, 2020

Published: December 29, 2020

Copyright $\odot 2020$ by author(s) and Scientific Research Publishing Inc. This work is licensed under the Creative Commons Attribution International License (CC BY 4.0).

http://creativecommons.org/licenses/by/4.0/

(c) (i) Open Access

\begin{abstract}
The paper discusses the researches that formed the basis of the study of the transition of "ordering-phase separation" and the reasons for such transition occurrence. Experimental results have presented what diffusion pairs are and how they occur in binary and multicomponent alloys. The paper illustrates that the chemical bonds between atoms are realized on the principle of pair interaction in both solid and liquid states of the alloy. The process of separating a multi-component $\mathrm{ABC}$ alloy into diffusion pairs $\mathrm{A} / \mathrm{B}, \mathrm{A} / \mathrm{C}$, and $\mathrm{B} / \mathrm{C}$ occurs in a liquid solution, where the diffusion mobility of atoms is very high, and the resistance of the environment is relatively low. The driving force of such a process is the chemical attraction between like and unlike atoms, that is, the tendency to phase separation and the tendency to ordering. Quenching the liquid alloy into the water fixes a microstructure consisting of microscopic areas corresponding in composition to one or another diffusion pairs. The paper shows what exactly should be done so that such a branch of science as Materials Science could get rid of the empirical approach when creating new alloys.
\end{abstract}

\section{Keywords}

Ordering-Phase Separation, Transition, Ionic Bond $\leftrightarrow$ Covalent Bond,

Pair Interaction, Diffusion Pairs, Transmission Electron Microscopy, Superalloys

\section{Introduction}

The contradictions discovered in the study of phase transformations in alloys were first seriously considered way back in the 1970s. The discussion held at that time on the initiative of Acta Metallurgica [1]-[6] did not lead to anything that could reasonably explain the appearance of particles of the second phase after quenching alloys from the solid solution range. However, this does not mean 
that such a discussion was useless. On the contrary, it helped to understand that an error crept somewhere in our ideas regarding diffusion phase transitions.

Moreover, later this error was found. The bottom line was that when interpreting data of X-ray phase analyses, no one paid attention to the existing limitations of the X-ray method. This method does not allow registering particles of a new phase if their size is smaller than the X-ray coherent scattering regions. At one time, A. Guinier [7] forewarned of these limitations. However, despite this warning, almost all phase diagram compilers utilized the X-ray phase analysis method without any doubt. When they have not detected particles of the second phase in the alloy structure they argued that such structure was a disordered solid solution. At the same time, it has been established that the resolution of the particles of the precipitated phase while using the transmission electron microscopy method (TEM) is by several orders higher than when using X-ray phase analysis. TEM allows identifying such fine particles at a resolution where the $\mathrm{X}$-ray phase analysis is powerless to compete.

The "ordering-phase separation" transition occurs in many binary alloys. It lies in the fact that at a quite certain temperature for every alloy, a seemingly surprising phenomenon occurs-the sign of the chemical interaction between dissimilar atoms changes. Consequent to such change, the microstructure type also changes: the previous microstructure dissolves and a microstructure forms corresponding to the new sign of the chemical interaction energy. Such transitions existence in nature means that all previous ideas about a solid solution, and its decomposition that occurred supposedly due to a decrease in solubility while lowering the temperature, are not consistent with reality [8].

For the first time, the "ordering-phase separation" transition was experimentally detected in alloys of the Fe-Cr system [9] by TEM. This transition in various binary alloys has definite characteristic features that become apparent at the level of microstructure changes: the sign of the energy of the chemical interaction between dissimilar atoms changes for each system at a specific temperature (the temperature of the transition of the "ordering-phase separation"). The microstructure that existed before the transition, for example, due to the tendency of the alloy to ordering (a solid solution with particles of chemical compounds) dissolves, and a microstructure forms in its place thanks to the tendency to phase separation (a solid solution with clusters or particles of the dissolved component). In some systems, such change in signs (and therefore the change in microstructure) can happen at more than at one temperature. When the energy of the chemical interatomic interaction turns out to be zero at the transition temperature, the microstructure of the alloy can have the form of a homogeneous solid solution (for example, in the Fe-Cr system). However, ranges of a uniform solid solution located between the phase separation areas and the areas of ordering were detected not all systems where such a transition occurred. In some cases, there was no sharp distinction, and particles of phases formed simultaneously on the same areas of the foil: ones-as a result of the tendency to ordering and others-as a result of phase separation [10]. 
A total absence of homogeneous solid solutions, detected by TEM in these alloys, indicates that all phase diagrams which represent these areas are based on incorrect interpretations of experimental results obtained using X-ray phase analysis. In this case, "the solid solutions range" quenching seems to be a completely unnecessary operation. In some cases, a disordered solid solution was not detected even on the "ordering-separation" transition line. Authors [11] studying the $\mathrm{Ni}$-Co system faced the situation when particles of the phase separation and particles of the ordering phases were detected near the transition temperature at the same time. Such a transition was called a diffuse "ordering-phase separation" transition.

The reasons for the attraction between dissimilar atoms, which lead to the formation of chemical compounds in alloys (tendency to ordering), and the reasons for the attraction between similar atoms, which lead to the formation of clusters or particles of atoms of the dissolved components (tendency to phase separation), have found now an adequate explanation in the framework of the existing electronic theory [12]. Assuming that a $100 \%$ metallic bond exists only in pure metals while in alloys, a certain part of valence electrons participate in the formation of other components of a strong chemical bond, the author [12] analyzed what kind of bonds exist between components using electronic theory. He came to the conclusion that the ionic bond arises in alloys when one or more pairs of valence electrons that, were supposed to participate in the formation of an electron gas are localized on the two nearest opposite atoms, leading to the formation of common orbitals in them. In this case, the formation of chemical compounds takes place in the alloy structure due to the attraction between the two nearest dissimilar neighbors. A covalent bond is formed when one or more pairs of valence electrons are involved in the formation of hybridized orbitals between two similar atoms. In this case, clusters or particles of the dissolved component are formed in the alloy structure due to the attraction between two similar atoms. This means that the property of alloys to change the sign of the chemical bond when the heating temperature changes, is derived from the very essence of the electronic structure of alloys [12].

This paper brings up an issue of how the formation of two-component phases occurs in multicomponent alloys under the conditions of paired chemical interaction between the nearest neighbors.

\section{Materials and Methods}

To answer the question of what structure the alloy has in the liquid state, the studied alloys were quenched by pouring out a small amount of liquid metal directly from the ladle into the water. We supposed that pouring out the metal not into any intermediate container, but directly into the water, would cast aside any doubts that we are fixing the microstructure of the liquid state in the alloy. Four binary alloys- $\mathrm{Co}_{3} \mathrm{~V}, \mathrm{Ni}_{68} \mathrm{Cr}_{32}, \mathrm{Ni}_{46} \mathrm{Cr}_{54}$ and $\mathrm{Ni}_{40} \mathrm{Cr}_{60}$-and the ternary alloy $\mathrm{Co}_{53} \mathrm{Mo}_{35} \mathrm{Cr}_{12}$ were used as examples for the study the latter is close in composition to the well-known Tribaloy alloys based on Co-E400C and E-800. They 
were melted in a vacuum induction furnace. Blanks for thin foils were cut from the obtained castings and foils were made using standard procedure. The study was carried out on an EM-125 electron microscope at an accelerating voltage of $100 \mathrm{kV}$ using standard procedure.

\section{Results and Discussion}

\subsection{Binary Alloys}

Previously, the author of this paper found that after water-quenching the $\mathrm{Co}_{3} \mathrm{~V}$ alloy from $1550^{\circ} \mathrm{C}$, there were found the rounded form particles of vanadium atoms in the microstructure of the alloy [13]. Detection of crystalline particles of a more refractory component in the structure of samples quenched from high temperatures was explained previously by the fact that such particles "did not have time to dissolve" at this temperature. We decided to verify this explanation. We melted the same alloy as in [13] and kept it at $1550^{\circ} \mathrm{C}$ for 2 hours, and after that, it was quenched into the water. Figure 1 shows the microstructure of this alloy. It turned out that the microstructure of the alloy quenched in water from $1550^{\circ} \mathrm{C}$ at no exposure and at 2 hours exposure are identical. This means that precisely this phase separation microstructure is equilibrium for the liquid state of the alloy at $1550^{\circ} \mathrm{C}$. Neither precipitates in the microstructure nor are satellites found near the matrix reflections at some microscopic distance from that point of the foil which structure is shown in Figure 1. A microstructure is visible that at initial approximation can be characterized as a section of a solid solution. This arrangement of vanadium particles in the microstructure suggests that they were precipitated into the liquid alloy in the first place than the rest of the matrix.

An even more interesting picture appeared in the $\mathrm{Ni}-\mathrm{Cr}$ system. After quenching the $\mathrm{Ni}_{40} \mathrm{Cr}_{60}$ and $\mathrm{Ni}_{68} \mathrm{Cr}_{32}$ alloys from $1450^{\circ} \mathrm{C}$ in water, two types of particles are fixed in the microstructure. In the $\mathrm{Ni}_{40} \mathrm{Cr}_{60}$ alloy, we can observe elongated $\mathrm{Ni}_{2} \mathrm{Cr}$ particles with boundaries consisting of a dense network of dislocations (Figure 2) as a consequence of the tendency towards ordering. In the $\mathrm{Ni}_{68} \mathrm{Cr}_{32}$ alloy, the rounded dark clusters of chromium atoms (with diffuse edges)

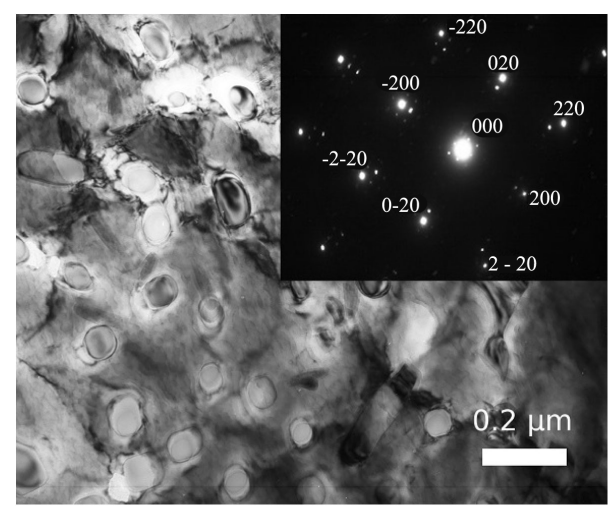

Figure 1. $\mathrm{Co}_{3} \mathrm{~V}$ alloy. Quenching into the water from a liquid state (2 hours holding at $\left.1550^{\circ} \mathrm{C}\right)$. Inset: Electron diffraction pattern. Vanadium particles inside a diffusion pair. 
as a consequence of the tendency to phase separation are observed (Figure 3). These results allowed the author [14] to conclude that the "ordering-phase separation" transition occurs not only when the temperature of the alloy changes but also when its composition changes.

In Figure 4, we were able to fix the boundary between the two types of microstructure-the structure of $\mathrm{Cr}$ clusters formed due to the tendency to phase separation (Figure 3 ) and $\mathrm{Ni}_{2} \mathrm{Cr}$ particles formed due to the tendency to ordering (Figure 2). In contrast to alloys of the $\mathrm{Fe}-\mathrm{Cr}$ system where regions of a disordered solid solution are located between these types of microstructure, these types of microstructure are in contact in alloys of the Ni-Cr system (Figure 4). Possibly, it was exactly this feature of the Ni-Cr alloys that previously led the

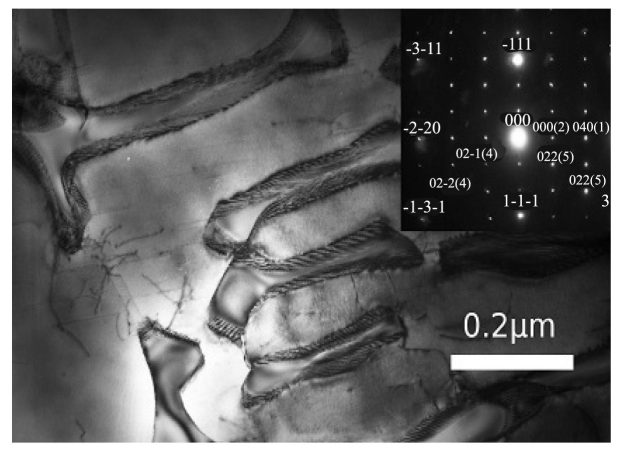

Figure 2. $\mathrm{Ni}_{40} \mathrm{Cr}_{60}$ alloy. Quenching into the water from a liquid state $\left(1450^{\circ} \mathrm{C}\right)$. Microstructure. Inset: Electron diffraction pattern. The particles of the $\mathrm{Ni}_{2} \mathrm{Cr}$ phase inside a diffusion pair.

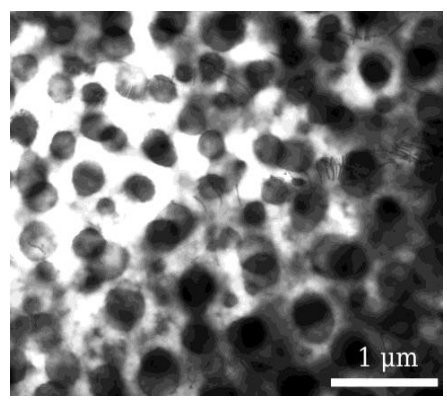

Figure 3. $\mathrm{Ni}_{68} \mathrm{Cr}_{32}$ alloy. Quenching into the water from a liquid state $\left(1450^{\circ} \mathrm{C}\right)$. Microstructure. Clusters of $\mathrm{Cr}$ atoms inside $\mathrm{Cr} / \mathrm{Ni}$ a diffusion pairs.

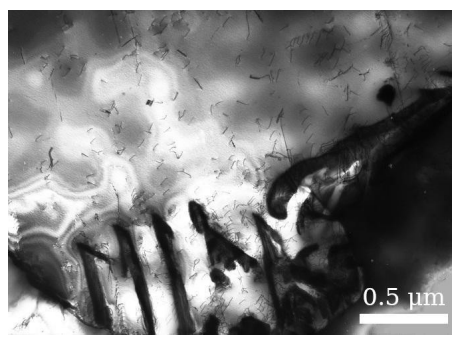

Figure 4. $\mathrm{Ni}_{46} \mathrm{Cr}_{54}$ alloy. Quenching into the water from a liquid state $\left(1450^{\circ} \mathrm{C}\right)$. Microstructure. The boundary between two diffusion pairs: in one of them, there is a tendency towards phase separation (above), in the other, towards ordering. 
author to conclude that the "ordering-phase separation" transition in these alloys can occur in different $\mathrm{Ni}-\mathrm{Cr}$ alloys at different temperatures [15].

The detection of a microstructure that is not a disordered solid solution after quenching from a liquid state (Figures 1-4) may indicate that chemical interactions exist in both crystalline and liquid states. This fact does not contradict the electronic theory of alloys.

Under the conditions of paired interactions existence, it is not difficult for us to understand the process of the formation of the chemical compounds (during ordering) or clusters (during phase separation) in binary alloys. At the same time, when such a process occurs in a three-component alloy it is hard to imagine how this can happen, if not to assume that particles of the three-component phases are formed in them. Apparently, this is precisely the reason why many researchers concluded that there is a possibility of precipitation of three-component Laves phases in ternary alloys, even though the electronic theory of alloys does not allow this possibility.

\section{2. $\mathrm{Co}_{53} \mathrm{Mo}_{35} \mathrm{Cr}_{12}$ Superalloy}

The experimental results obtained by using TEM on the three-component $\mathrm{Co}_{53} \mathrm{Mo}_{35} \mathrm{Cr}_{12}$ alloy [16] show that only two-component chemical compounds as well as two-component clusters or one-component particles of a dissolved element can be detected in them [8]. Because covalent and ionic bonds arise in alloys thanks to pairwise interactions between the nearest neighbors, three-component phases cannot be formed in principle. The following question immediately arises: how should the redistribution of atoms of each of the three components occur so that the two-component phase can form from a disordered solution? As noted above, when a binary alloy is in a liquid state, because of the very high diffusion rate the atoms of each of the components reach the equilibrium distribution of the chemical potential throughout the entire volume of the alloy easily enough. This means that at such a diffusion rate in the $A B C$ alloy sections of the alloy with approaching in composition to $\mathrm{AB}, \mathrm{AC}$, or $\mathrm{BC}$ pairs are formed easily if there is a tendency to ordering in these pairs. The pairs $\mathrm{AA}, \mathrm{BB}$, and $\mathrm{CC}$ are formed if there is a tendency to phase separation. If such a liquid alloy is quenched into the water, then the structure of such an equilibrium state can be revealed.

Unfortunately, the concept of the "diffusion pair" has not yet been included in the everyday life of materials scientists since there is no convincing experimental evidence of its real existence. The present author introducing the concept of a diffusion pair tried to prove that the formation of such pairs occurs precisely in molten metal [8]. When the alloy solidifies, the structure consisting of such diffusion pairs is preserved, and corresponding phase particles can be formed inside of such pairs. All these explanations are like fantasy since we are used to believing that there are no chemical bonds in alloys at high temperatures. It is believed that the mutual arrangement of atoms of different types is completely chaotic in the liquid state and that chemical bonds "arise" only at relatively low 
heating temperatures.

Alloys of such composition as $\mathrm{Co}_{53} \mathrm{Mo}_{35} \mathrm{Cr}_{12}$ superalloy belong to a group of wear-resistant alloys, which, as it is considered [17], obtains high strength and hardness due to the formation of coarsely dispersed intermetallic Laves phase particles. Its composition is similar to that of known Cobalt-based Tribaloy alloys-T-400C and T-800, containing approximately the same amount of Mo and $\mathrm{Cr}$ as in the $\mathrm{Co}_{53} \mathrm{Mo}_{35} \mathrm{Cr}_{12}$ alloy [17]. Alloys of this composition are generally regarded as hyper eutectic alloys, containing $30 \%-70 \%$ of intermetallic distributed in a relatively soft solid solution [18]. The solid primary phase is a three-component Laves phase having a melting point of about $1560^{\circ} \mathrm{C}$ and a composition approximately corresponding to compositions of $\mathrm{Co}_{3} \mathrm{Mo}_{2} \mathrm{Si}$ or CoMoSi [18].

Figure 5(a) shows a bright-field image of the microstructure of the $\mathrm{Co}_{53} \mathrm{Mo}_{35} \mathrm{Cr}_{12}$ alloy after its quenching in water from a liquid state $\left(1550^{\circ} \mathrm{C}\right)$. Alternating dark and light elongated grains of the $\mathrm{Co}_{3} \mathrm{Mo}$ phase can be observed in the form of slightly flexed arcs. Inside light arcs we can observe narrow transverse dark bands, which occur in the crystal lattice during quenching. It is apparent, that these particles of the $\mathrm{Co}_{3} \mathrm{Mo}$ phase are located inside the $\mathrm{Co} / \mathrm{Mo}$ diffusion pair. Figure $5(\mathrm{~b})$ shows the electron diffraction pattern taken from the microstructure in Figure 5(a). The pattern indicates that such particles have the hexagonal structure $\mathrm{D}_{19}$, which is characteristic of the $\mathrm{Co}_{3} \mathrm{Mo}$ phase. These grains form at $1340^{\circ} \mathrm{C}$, i.e. at the moment when the $\mathrm{Co}_{3}$ Mo phase solidifies. Along with reflections from a chemical compound, randomly scattered reflections are observed apparently from $\mathrm{Cr}$ particles.

In Figure 6, we presented the location of diffusion pairs and binary phases in the $\mathrm{Co}_{53} \mathrm{Mo}_{35} \mathrm{Cr}_{12}$ ternary alloy at a temperature above the melting point of the alloy. Dashed lines indicate hypothetical boundaries of diffusion pairs. Inside these diffusion pairs, precipitates 3 ( $\mathrm{Co}_{3} \mathrm{Mo}$ chemical compound) or 4 (clusters or particles of $\mathrm{Cr}$ atoms) are formed. Space 5 marks the "boundary" separating microscopic sites 1 and 2 .

Figure 7 shows the cellular microstructure which was formed due to the tendency to phase separation at $1300^{\circ} \mathrm{C}$ in the $\mathrm{Co}_{70} \mathrm{Mo}_{30}$ binary alloy [19]. This alloy has almost the same amount of Mo as the three-component alloy under study.

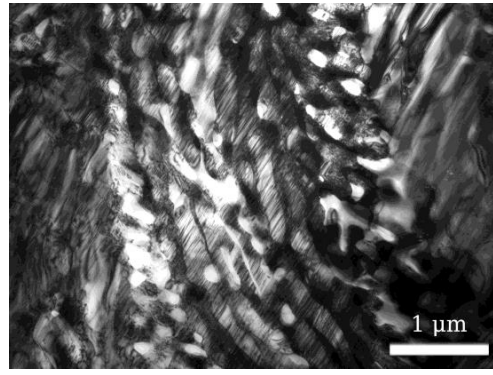

(a)

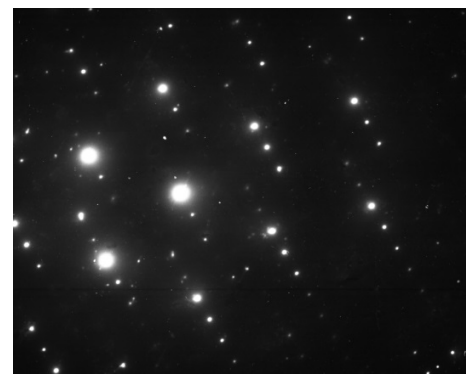

(b)

Figure 5. $\mathrm{Co}_{53} \mathrm{Mo}_{35} \mathrm{Cr}_{12}$ alloy. Quenching into the water from liquid state $\left(1550^{\circ} \mathrm{C}\right)$. (a) Microstructure; (b) Electron diffraction pattern. 


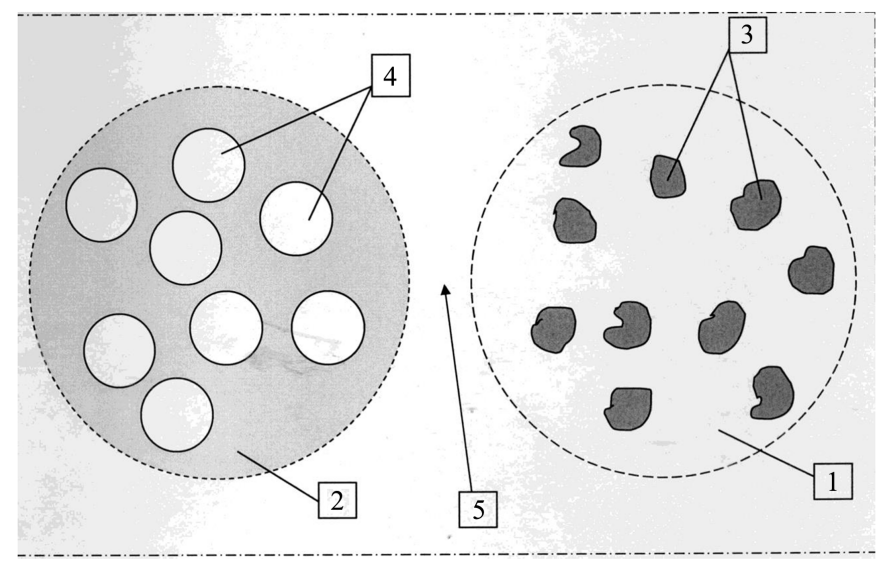

Figure 6. An image of diffusion pairs and phases in $\mathrm{Co}_{53} \mathrm{Mo}_{35} \mathrm{Cr}_{12}$ alloy.

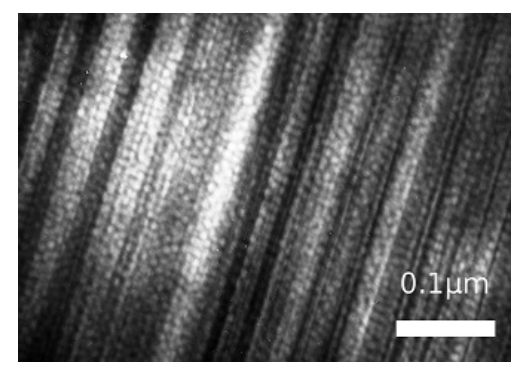

Figure 7. $\mathrm{Co}_{70} \mathrm{Mo}_{30}$ binary alloy. Quenching into the water from $1300^{\circ} \mathrm{C}$. Microstructure.

Molybdenum atoms are present in the dark boundaries of the cells, and cobalt atoms are present in the cell body. That design of the cells and their size determine the very high hardness $(800-850 \mathrm{HV})$ and satisfactory ductility of the alloy researched. This shows that the "ordering-phase separation" transition occurs in the $\mathrm{Co}_{70} \mathrm{Mo}_{30}$ binary alloy somewhere in the temperature range between solidus and $1300^{\circ} \mathrm{C}$.

However, in the $\mathrm{Co}_{53} \mathrm{Mo}_{35} \mathrm{Cr}_{12}$ alloy instead of the cellular structure stacking faults are observed when quenched from $1300^{\circ} \mathrm{C}$ (Figure 8). If we look at the generally accepted phase diagrams of $\mathrm{Co}-\mathrm{Cr}$ and $\mathrm{Cr}-\mathrm{Mo}$ we can conclude that with approximately the same component ratios as in the corresponding diffusion pairs of the $\mathrm{Co}_{53} \mathrm{Mo}_{35} \mathrm{Cr}_{12}$ alloy under study, only a tendency to phase separation takes place between $\mathrm{Co}$ and $\mathrm{Cr}$ atoms, $\mathrm{Cr}$ and $\mathrm{Mo}$ atoms, in the entire range of heating temperatures. So, chromium prevents the formation of a cellular microstructure when it is added in the binary alloy $\mathrm{Co}_{70} \mathrm{Mo}_{30}$. This can have a negative effect on the strength and ductility of the alloy at high temperatures. This fact indicates that chromium in the alloy under study plays the role of harmful impurities. Other small additives, for example, silicon and carbon in Tribaloy alloys (T-400C and T-800) [18] can be considered also as harmful impurities.

The question arises immediately: why does chromium prevent the formation of a cellular structure? It would seem that chromium cannot affect the chemical interactions in the $\mathrm{Co} / \mathrm{Mo}$ diffusion pair. However, it should not be forgotten that chromium affects the structure in $\mathrm{Co} / \mathrm{Cr}$ and $\mathrm{Mo} / \mathrm{Cr}$ diffusion pairs, and 


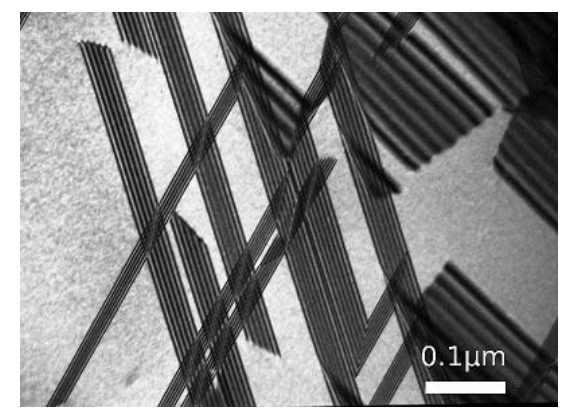

Figure 8. $\mathrm{Co}_{53} \mathrm{Mo}_{35} \mathrm{Cr}_{12}$ alloy. Water quenching from $1300^{\circ} \mathrm{C}$. Microstructure.

therefore, it can change the quantitative ratios of elements in the Co/Mo diffusion pair and instead of cells lead to the formation of stacking faults in the microstructure. Therefore, any addition to a multi-component (super) alloy must be substantiated in full.

It is worth bearing in mind that attempts to achieve higher properties of alloys (superalloys, high-entropy alloys, etc.) that use old methods and approaches, for example, by complicating their chemical composition, are unlikely to lead to improvement of their properties in conditions pair interatomic interaction. Simplifying the composition of, for example, some superalloys, will make it possible to full use of the heat-resistant capabilities of the main pair of components of a particular alloy.

So, as shown by TEM the decomposition of alloys begins in a liquid state through the diffusion pair formation. If the melting point of the phase is higher than the temperature of the molten metal, the simultaneous formation of the diffusion pairs and particles of a new phase inside these pairs is possible (for example, in the $\mathrm{Co}_{53} \mathrm{Mo}_{35} \mathrm{Cr}_{12}$ alloy). As the temperature of the alloy decreases, the decomposition process continues in each type of diffusion pairs in its way. In a pair where there is a tendency to ordering, particles of the corresponding chemical compound are formed. In a pair where there is a tendency to phase separation, particles (clusters) of a corresponding solute are formed. If in any binary alloy, the "ordering-phase separation" transition occurs with a change in temperature then the same transition will also occur in diffusion pair close to it in composition and temperature. So, as shown by TEM the decomposition of alloys begins in a liquid state through the diffusion pair formation. If the melting point of the phase is higher than the temperature of the molten metal, the simultaneous formation of the diffusion pairs and particles of a new phase inside these pairs is possible (for example, in the $\mathrm{Co}_{53} \mathrm{Mo}_{35} \mathrm{Cr}_{12}$ alloy). As the temperature of the alloy decreases, the decomposition process continues in each type of diffusion pairs in its way. In a pair where there is a tendency to ordering, particles of the corresponding chemical compound are formed. In a pair where there is a tendency to phase separation, particles (clusters) of a corresponding solute are formed. If in any binary alloy, the "ordering-phase separation" transition occurs with a change in temperature then the same transition will also occur in diffusion pair close to it in composition and temperature 
Sizes and composition of diffusion pairs preserve until the next re-melting, regardless of whether an alloy was subjected to heat treatment when it was in a crystalline state. With a change in the heat treatment temperature only the microstructure inside each type of diffusion pair changes.

\section{Conclusions}

Introduction to such a concept as a "diffusion pair" completely changes our way of thinking about how the process of the nucleation of new phases in alloys, including in multi-component, occurs and why the precipitated phases can only be one- or two-component, but not multi-component.

It was experimentally found that the starting point for the decomposition of an alloy is not the formation of a disordered solid solution after quenching, but the liquid state the alloy is in during its smelting, when in it diffusion pairs are formed thanks to the existence of ionic and covalent chemical bonds between the nearest neighbors. The initial structure for the decomposition of the alloy is the microstructure, which is fixed by quenching in water from a liquid state (structure of diffusion pairs).

A structure of diffusion pairs comprises microscopic sites, each of which represents one or other binary micro-solution enriched by atoms of one solute. Theoretically, a multi-component alloy in the course of its smelting is divided into as many types of diffusion pairs as its composition allows. Heat treatment at various temperatures below the melting point leads to the formation inside diffusion pairs of particles of two types. These are chemical compounds (tendency to ordering) or two-component clusters and particles comprising atoms of one component (tendency to phase separation).

This research did not receive any specific grant from funding agencies in the public, commercial, or not-for-profit sectors.

\section{Conflicts of Interest}

The author declares no conflicts of interest regarding the publication of this paper.

\section{References}

[1] Woodilla, J.E. and Averbach, B.L. (1968) Modulated Structures in Au-Ni Alloys. Acta Metallurgica, 16, 255-263. https://doi.org/10.1016/0001-6160(68)90121-1

[2] Chevalier, J.P. and Stobbs, W.M. (1976) An Electron Diffraction Study of Short-Range Order in Quenched $\mathrm{Ni}_{4}$ Mo Alloys. Acta Metallurgica, 24, 535-542. https://doi.org/10.1016/0001-6160(76)90098-5

[3] Higgins, J., Nicholson, R.B. and Wilkes, P. (1974) Precipitation in Iron-Beryllium System. Acta Metallurgica, 22, 201-217. https://doi.org/10.1016/0001-6160(74)90011-X

[4] Laughlin, D.E. and Cahn, J.W. (1975) Spinodal Decomposition in Age-Hardening Cu-Ti Alloys. Acta Metallurgica, 23, 329-339.

https://doi.org/10.1016/0001-6160(75)90125-X 
[5] Phillips, L.A. and Tanner, L.E. (1973) High-Resolution Electron Microscopy Observations on GP Zones in an Aged Cu-2 wt.\% Be Crystal. Acta Metallurgica, 21, 441-448. https://doi.org/10.1016/0001-6160(73)90201-0

[6] Flewitt, P.E. (1974) Phase Transformations in Nb 16 to $40 \% \mathrm{Zr}$ Alloys above the Monotectoid Temperature. Acta Metallurgica, 22, 47-63. https://doi.org/10.1016/0001-6160(74)90125-4

[7] Guinier, A. (1963) X-Ray Diffraction in Crystals, Imperfect Crystals, and Amorphous Bodies. WH1 Freeman, San Francisco.

[8] Ustinovshikov, Y. (2019) The "Ordering-Phase Separation" Transition in Alloys". Monograph. Manchester Scholars Publishing, Great Britain.

[9] Ustinovshikov, Y., Shirobokova, M. and Pushkarev, B. (1996) A Structural Study of the Fe-Cr System Alloys. Acta Materialia, 44, 5021-5032. https://doi.org/10.1016/S1359-6454(96)00088-2

[10] Ustinovshikov, Y. (2020) Chemical Bonds in Metallic Alloys. International Journal of Materials Research in Science \& Technology, 1, 35-45. https://doi.org/10.47890/IJMRST/2020/YUstinovshikov/14205435

[11] Ustinovshikov, Y., Shabanova, I. and Lomova, N. (2013) TEM Study of the 'Ordering-Phase Separation' Transition in Ni-Co Alloys. Journal Advanced Microscopy Researches, 8, 276-282. https://doi.org/10.1166/jamr.2013.1170

[12] Ustinovshikov, Y. (2017) Changes in Electronic Structure of Metallic Alloys at the Transition 'Ordering-Phase-Separation'. Journal of Alloys and Compounds, 714, 476-483. https://doi.org/10.1016/j.jallcom.2017.04.250

[13] Ustinovshikov, Y. (2015) Phase Transition Ordering-Phase Separation in the $\mathrm{Co}_{3} \mathrm{~V}$ Alloy. Journal of Alloys and Compounds, 639, 669-674.

https://doi.org/10.1016/j.jallcom.2015.03.180

[14] Ustinovshikov, Y. (2012) Phase Transformations in Alloys of the Ni-Cr System. Journal of Alloys and Compounds, 547C, 227-232.

https://doi.org/10.1016/j.jallcom.2012.05.120

[15] Ustinovshikov, Y. (2015) Phase Transition "Ordering-Phase Separation" in Alloys of Ni-Cr System. Materials Chemistry and Physics, 165, 14-18.

https://doi.org/10.1016/j.matchemphys.2015.05.027

[16] Ustinovshikov, Y. (2014) Structural Transformations in the $\mathrm{Co}_{53} \mathrm{Mo}_{35} \mathrm{Cr}_{12}$ Alloy at Different Temperatures. Journal of Alloys and Compounds, 602, 135-139. https://doi.org/10.1016/j.jallcom.2014.02.135

[17] Day, J., Xuang, X. and Yao, M. (2004) Study of Composition-Induced Microstructure Variation in the Interface between Co-Based Hard Facing Alloy and IN738 Ni-Based Superalloy. Journal of Materials Engineering and Performance, 13, 158-166. https://doi.org/10.1361/10599490418352

[18] Nsoesie, S., Liu, R., Jiang, K. and Liang, M. (2013) High-Temperature Hardness and Wear Resistance of Cobalt-Based Tribaloy Alloys. International Journal of Mechanical and Materials Engineering, 2, 48-56.

[19] Ustinovshikov, Y. and Shabanova, I. (2012) Nature of High Hardness in the $\mathrm{Co}_{70} \mathrm{Mo}_{30}$ Alloy. Materials Chemistry and Physics, 135, 254-258.

https://doi.org/10.1016/j.matchemphys.2012.04.046 